\title{
Bullying still rife in medical training
}

$\mathrm{D}$ r. Christopher Lemieux felt helpless the first time he witnessed a supervising physician humiliate a fellow resident.

"The doctor was reviewing a consult with the resident and just ripped it up, telling them in front of everybody that it would be easier to do it again himself than to correct the consult," recalls Lemieux, now secretary of the Fédération des médecins résidents du Québec (FMRQ). "I wasn't able to do anything and the resident didn't do anything."

For years, many residents across the country have reported similar experiences. FMRQ and Resident Doctors of Canada (RDoC) first called attention to widespread harassment and intimidation of medical trainees in 1996. At that point, residents reported "no effective or enforceable policy to deal with this problem in any jurisdiction." Medical schools, residency programs and accrediting bodies responded, introducing policies and programs to address complaints and support the overall wellbeing of trainees and faculty. Two decades on, rates of bullying remain high, says Lemieux, likening the problem to a resistant infection.

Some $73 \%$ of residents surveyed by RDoC in 2012 said they were exposed to inappropriate behaviour during training that made them feel diminished. Other surveys show similarly high rates of intimidation and harassment, with $45 \%-93 \%$ of residents reporting at least one experience. The mistreatment comes from all corners: in a 2008 survey, $55 \%$ of residents reported being bullied by nurses, $45 \%$ by patients, $42 \%$ by supervising physicians and $26 \%$ by other residents.

Lemieux and others share stories of surgeons throwing medical instruments at trainees who make mistakes, of nurses belittling or ignoring residents' clinical decisions, of pressure to disobey restrictions on working hours, of yelling, threats and sleazy comments, among other abuses. RDoC reports that about a third of these incidents are never officially reported.

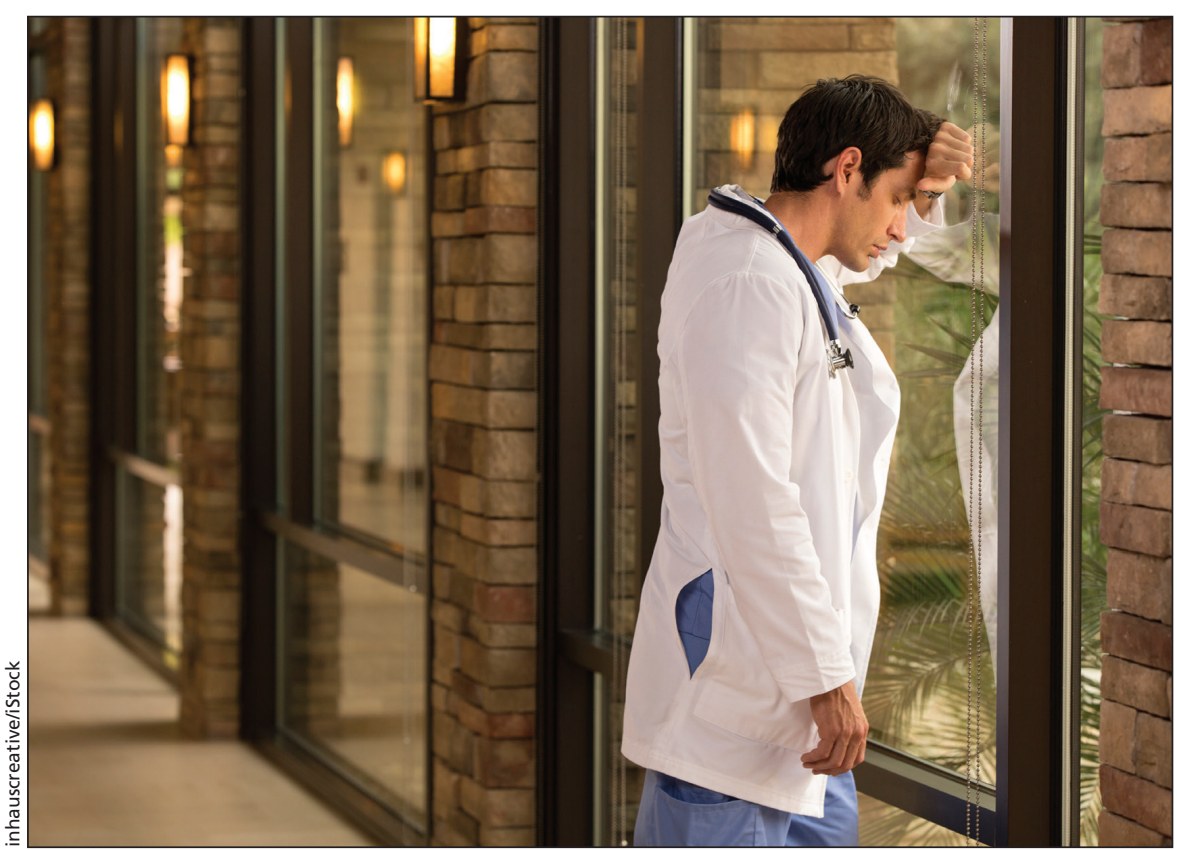

Medical students and residents continue to face high rates of intimidation and harassment despite efforts to counter abuse, say trainee groups.

Dr. Breanna Balaton, cochair of RDoC's wellness committee, says the problem is no longer a lack of procedures to address abuse. "If you look hard enough, there are a lot of resources: through your program and postgraduate medical education office, the wellness offices with people who will keep these things confidential and the provincial health staff organizations."

Now, the main problem is that residents still don't know or trust these resources, she explains. RDoC's 2013 national survey showed that $33 \%$ of respondents believed reporting would not have changed the situation, $21 \%$ didn't do anything for fear of reprisal, $13 \%$ identified confidentiality as a concern and $11 \%$ were unaware of reporting procedures.

Some residents also rationalized the abuse as an unavoidable, even necessary evil of medical education, Balaton adds. "If that's what we're seeing, then that's what we pick up and how we go on to teach others."

According to Marie-Pier Bastrash, vice president of student affairs for the
Canadian Federation of Medical Students (CFMS), these beliefs are often set during undergraduate medical education. "We're working in teams with residents who work with staff, so often the same issues of humiliation and belittlement come up."

CFMS, RDoC and FMRQ each have initiatives underway to educate medical students and residents about the forms of recourse available to them. These initiatives include developing simple guides to reporting abuse at medical schools and residency programs across Canada. The groups will also publish the results of new wellness surveys of their memberships in coming months and are updating recommendations for reducing abuse.

FMRQ released a new position paper on Jan. 27, which recommends reinforcing the positive behaviour of physicians who model collegiality, and is planning a social media campaign to celebrate these roles models, says Lemieux.

According to Dr. Glen Bandiera, associate dean of postgraduate medical education at the University of Toronto, the change to a culture of zero-tolerance 
for bullying in medicine will ultimately be "evolutionary as opposed to revolutionary."

"The new incoming cohort of students and residents over the last few years are more willing to identify these issues, and are more willing to exercise the options available to them," he says. "That also bears out in faculty over time: as people have experienced different ways of learning, the old environment is gradually going to disappear."
Canada. "We've taken a very aggressive stance on reviewing programs ... and we're currently rewriting the standards of accreditation in Canada to increase their rigor," he explains. "There's going to be a new standard, called the learners, teachers and administrators' standard, and it has a lot to do with the positivity of the learning experience."

The degree to which residency programs can "demonstrate concrete positive outcomes to residents, that's proba-

\section{"There's going to be a new standard... and it has a lot to do with the positivity of the learning experience."}

Top-down measures, such as stronger accreditation standards requiring medical schools and residency programs to have effective mechanisms for addressing abuse, are part of that evolution, says Bandiera, who chairs the committee on accreditation for the Royal College of Physicians and Surgeons of bly the most important thing," Bandiera adds. This is often complicated by the need to preserve confidentiality of investigations, even after they've been resolved, he says. "Sometimes the perception may be nothing is happening, when in fact a lot of work may be happening in the background."
Medical schools and residency programs are also making it easier and safer for trainees to log complaints through web-based reporting tools and options to flag abuse anonymously, among other strategies. At the University of Toronto, for example, residents have "multiple routes to access the system, whether through a direct supervisor, a rotation coordinator, a program director or a third party," says Bandiera.

Dr. Armand Aalamian, associate dean of postgraduate medical education at McGill University in Montréal, says preventing abuse will also require putting more resources into supports for trainees and faculty. "We're all dealing with a system, in Quebec and across Canada, that is under quite a bit of stress in terms of budget cuts, in terms of working hours, in terms of resources, so when people are stressed that's when you have issues of behaviour conflicts." — Lauren Vogel, CMAJ

CMAJ 2016. DOI:10.1503/cmaj.109-5237

\section{Drug-resistant tuberculosis plagues India}

\section{Part one of a series}

'I ndia is unlucky to be lucky." This non sequitur, repeated by health care providers in both Delhi and Mumbai, India, was invariably followed by a pause for effect, then the explanation. India's 1.3 billion people have easy access to inexpensive drugs - often extraordinarily easy at neighbourhood pharmacy counters, where prescriptions are unnecessary. But this access is exacting a high price: drug resistance. Tuberculosis (TB) is a case in point.

Of the nine million reported cases of TB worldwide, 2.3 million are in India, according to the World Health Organization's (WHO) Global Tuberculosis Report 2015. That number is relatively stable (though it has decreased everywhere else, according to the report), but the number with multi-drug resistant TB (MDR-TB) is on the rise. In 2008, about 99000 people were newly diag- nosed or in retreatment for MDR-TB; by 2014 , that number had increased nearly four times, to about 395600 , or $17.2 \%$ of all TB patients.

"India is unlucky to be lucky," said Dr. Sylvie Jonckheere, the medical technical referent at the Médecins sans Frontière (MSF) clinic in Mumbai. In part, this is because drugs are inexpensive. India is the pharmacy to the developing world because its unique patent system allows it to legally manufacture and sell drugs at a fraction of the price charged in other countries. But the availability of inexpensive meds also means they are overused. For example, most of the firstline antitubercular drugs and fluoroquinolones are routinely available over-thecounter and used to treat minor illnesses.

"We have patients who have already been exposed to reconverted drugs so even with the new drugs, it's difficult to have an effective regime," said Jonckheere. "Women with urinary tract infections are taking drugs that are scary because the infections aren't sensitive to many things any more. Abuse of antibiotics is widespread. In this country you can get anything without a prescription." Things are improving somewhat these days, she adds: "You kind of have to say you're a doctor." Nevertheless, "we're creating a future nightmare for my future colleagues in the infectious diseases business I think."

Like other infectious diseases, at least four drugs are needed to treat TB. Jonckheere is seeing some TB that's resistant to 12 or 14 drugs.

According to WHO definitions, MDR-TB is resistant to isoniazid and rifampin - the two most potent and frequently used $\mathrm{TB}$ drugs - and may also be resistant to other first-line drugs. Extremely drug-resistant TB (XDR-TB) is resistant to isoniazid and rifampicin, and to any fluoroquinolone, as well as to any of the three second-line injectables (amikacin, capreomycin and kanamycin). While XXDR-TB is resistant to all first- 